\title{
Change of drift in one-dimensional diffusions
}

\author{
Sascha Desmettre ${ }^{1}$. Gunther Leobacher ${ }^{2}$. \\ L.C.G. Rogers ${ }^{3}$
}

Received: 25 October 2019 / Accepted: 25 November 2020 / Published online: 17 March 2021

(C) The Author(s) 2021

\begin{abstract}
It is generally understood that a given one-dimensional diffusion may be transformed by a Cameron-Martin-Girsanov measure change into another onedimensional diffusion with the same volatility but a different drift. But to achieve this, we have to know that the change-of-measure local martingale that we write down is a true martingale. We provide a complete characterisation of when this happens. This enables us to discuss the absence of arbitrage in a generalised Heston model including the case where the Feller condition for the volatility process is violated.
\end{abstract}

Keywords One-dimensional diffusions · Change of measure · Heston model · Feller condition $\cdot$ Free lunch with vanishing risk

Mathematics Subject Classification (2010) $60 \mathrm{~J} 60 \cdot 91 \mathrm{~B} 70$

\section{JEL Classification G13}

\section{Introduction}

Our original goal in this paper was to understand how a change of measure works in the celebrated stochastic volatility model of Heston [11]. When this model is specified, if the growth rate of the asset is not equal to the riskless rate, then we need to change measure to a pricing measure in which the growth rate $i s$ the riskless rate. The

$凶$ S. Desmettre

sascha.desmettre@jku.at

1 Institute of Financial Mathematics and Applied Number Theory, Johannes Kepler University Linz, Altenbergerstraße 69, 4040 Linz, Austria

2 Department of Mathematics and Scientific Computing, University of Graz, Heinrichstr. 36, 8010 Graz, Austria

3 Statistical Laboratory, University of Cambridge, Wilberforce Road, Cambridge CB3 OWB, UK 
question then arises: 'Can this be done?' The answer we found was 'Not always'; and in cases where it cannot be done, general results then say that there must be arbitrage (in a suitable sense).

We then realised that the question is closely related to changing the given drift of a one-dimensional diffusion to a different drift, using a change of measure. This uses the Cameron-Martin-Girsanov theorem; but as is well known, this very general result cannot be applied without care, the main point being to decide whether the local martingale we write down to do the change of drift is actually a martingale. In general, this is hard to decide; but in the special case that concerns us, where the drift is again a function of the diffusion, we are able to derive necessary and sufficient conditions for the change of measure to 'work'. We present this in Sect. 2, as an algorithm to be followed to decide for any particular situation, and we illustrate this with two interesting examples. It might appear that the result we give is a reprise of the main results of Mijatović and Urusov [17, 16], but what we do here is actually rather different. A standing assumption throughout $[17,16]$ is that if a boundary point of the diffusion can be reached in finite time, then the diffusion stops there. For the application we have in mind, in Sect. 3, this assumption does not hold; we want to consider CIR processes which reach zero and immediately return. Unusually, this behaviour can be completely specified by an SDE with nice coefficients; but more generally, a diffusion which can reflect from a boundary point cannot be specified by an SDE without explicitly involving a local time term, as in the Tanaka SDE for reflecting Brownian motion. While solutions of SDEs are very general regular onedimensional diffusions, they are not the most general examples; as is well known, the most general regular one-dimensional diffusion is specified by its scale function and speed measure, and for our purposes, it is necessary to work in this setting. This requires us to identify the Markov-process form of the Cameron-Martin-Girsanov change-of-measure local martingale, and to understand its effect on the generator of the diffusion. All this is explained in Sect. 2; we are discussing there the transformation of Markov processes by multiplicative functionals, a topic which goes back a very long way, to Itô and Watanabe [14], Dynkin [8] and references therein, and which is still of interest nowadays; see e.g. Palmowski and Rolski [18] or Çetin [3].

Our work shares common features with Hulley and Platen [12, 13] and Kotani [15], who also work with the scale and speed representation (the main result of [15] is also obtained by Delbaen and Shirakawa [7] using different methods). The question they answer is: If a one-dimensional diffusion $X$ is in natural scale, when is it a martingale, and not a local martingale? Our study determines when the changeof-measure local martingale $Z$ is a true martingale, which includes the problem of Hulley and Platen $[12,13]$ and Kotani [15] as the special case $Z=X$.

In Sect. 3, we turn to the Heston model for the stock price $S$ and the volatility $v$, which defines their evolution in the 'real-world' probability $P$ as

$$
\begin{aligned}
d S_{t} / S_{t} & =\mu\left(v_{t}\right) d t+\sqrt{v_{t}}\left(\rho d W_{t}+\rho^{\prime} d W_{t}^{\prime}\right), \\
d v_{t} & =\kappa\left(\theta-v_{t}\right) d t+\sigma \sqrt{v_{t}} d W_{t} .
\end{aligned}
$$

Here, $W$ and $W^{\prime}$ are independent Brownian motions, $\kappa, \theta$ and $\sigma$ are strictly positive constants, and $\rho \in(-1,1)$ is the constant correlation between the Brownian motions driving stock and volatility. We write $\rho^{\prime}:=\sqrt{1-\rho^{2}}$. The function $\mu$ is assumed con- 
tinuous. We refer to the model (1.1), (1.2) as the generalised Heston model; a special case appears in Heston's original paper and many other studies, where $\mu$ is taken to be constant, as is the riskless rate of interest $r$. Here we take $r=0$ throughout in order to simplify notation; this loses no generality, as we could equally consider $S$ defined by (1.1) to be the discounted stock $\left(e^{-r t} S_{t}\right)$.

In option pricing papers on Heston's stochastic volatility model, it is typically assumed that a risk-neutral measure $\tilde{P}$ exists and that the dynamics are stated in the corresponding risk-neutral form; see for example the extensive textbook Rouah [22, in particular Chap. 1] and the references therein. However, the question of existence of such a risk-neutral measure is rarely investigated - save for the trivial case $\mu=r$. But the absence of such a risk-neutral measure implies existence of free lunch with vanishing risk, that is, a form of arbitrage! A notable exception where this problem is addressed is Wong and Heyde [25], where the authors give a solution to this problem assuming the Feller condition

$$
\delta:=\frac{4 \kappa \theta}{\sigma^{2}} \geq 2
$$

a condition which keeps the volatility process strictly positive. This condition relates only to the diffusion for $v$; in particular, it is not related to what the drift $\mu$ may be.

However, the Feller condition is frequently violated in practice as has been pointed out in Albrecher et al. [1] or Clark [4, in particular Table 6.3]. Building on results in Mijatović and Urusov [16, 17], this problem is addressed for several stochastic volatility models, including the classical Heston model, in Bernard et al. [2] by modifying the model so that the volatility process is stopped as soon as it hits 0 . While it mathematically solves the problems incurred by a violated Feller condition, this approach is not convincing from an economic point of view.

In Sect. 3, we show that in the classical Heston model where the function $\mu$ is constant and non-zero, a failure of the Feller condition implies that there is no riskneutral measure. However, if the drift $\mu$ is not constant but satisfies a simple integrability condition at 0 , we show that there is an equivalent local martingale measure (ELMM) even in the case where the Feller condition is not satisfied. When the Feller condition is satisfied, we show that there is always an ELMM.

In the Appendix, as a gentle amusement, we directly construct a free lunch with vanishing risk (FLVR), from which it follows by the celebrated fundamental theorem of asset pricing (FTAP) of Delbaen and Schachermayer [5, 6] that there is no equivalent $\sigma$-martingale measure and a fortiori no ELMM.

Does it really matter if the Feller condition fails, so that there is no ELMM? It does not; all that has happened is that we started off from a bad place, and what we should do is to immediately put ourselves into the risk-neutral measure (in effect, assume that $\mu=0$ ). We gain nothing by being overly introspective about the growth rate of a stock, about which we know next to nothing in any case.

\section{Changing measure in a one-dimensional diffusion}

We begin with a regular diffusion taking values in an interval $I \subseteq \mathbb{R}$. The killing measure is assumed to be zero. We write $I^{\circ}$ for the interior of $I$, which could be 
equal to $I$. We also set $a=\inf I, b=\sup I$ for the endpoints of $I$. The interval $I$ may be the whole real line, and may contain endpoints or not. We write $C_{0}$ for the space of continuous functions $f: I \rightarrow \mathbb{R}$ with limits at the endpoints.

We let $\Omega=C\left(\mathbb{R}_{+} ; I\right)$ be the canonical path space with the canonical process $X_{t}(\omega)=\omega(t)$ and the raw filtration given by $\mathcal{F}_{t}^{0}=\sigma\left(X_{s}: s \leq t\right)$. If $P$ is the law of $X$ on $\left(\Omega, \mathcal{F}_{\infty}^{0}\right)$, we let $\left(\mathcal{F}_{t}\right)$ be the universal completion of $\left(\mathcal{F}_{t}^{0}\right)$. We write $\mathcal{F}=\mathcal{F}_{\infty}$ for brevity. We write $s$ for the scale function of $X$ and $m$ for its speed measure, so that the infinitesimal generator of $X$ is

$$
\mathcal{G}=\frac{1}{2} \frac{d^{2}}{d m d s}
$$

see for example Revuz and Yor [19, Theorem VII.3.12] (while noting the different scaling factor for the speed measure there). If a boundary point is in $I$ ( $a \in I$, to fix ideas), there is a boundary condition

$$
\frac{d f}{d s}(a+)=2 m(\{a\}) \mathcal{G} f(a) .
$$

The corresponding boundary condition, if $b \in I$, is $\frac{d f}{d s}(b-)=2 m(\{b\}) \mathcal{G} f(b)$. See [19, Proposition VII.3.13], again noting the different scaling factor there. We omit discussion of the situation $m(\{a\})=\infty$ or $m(\{b\})=\infty$, corresponding to absorption at the boundary, as this is a special case already dealt with in the earlier works of Mijatović and Urusov [16, 17]. Moreover, for volatility models which are our main application, absorbing boundaries are not reasonable from an economic point of view.

Specifying the domain of functions on which $\mathcal{G}$ acts is important. We fix some reference point $\xi \in I^{\circ}$ and define the domain $\mathcal{D}$ of $\mathcal{G}$ to be the set of all $f$ which are represented as

$$
f(x)=c_{1}+\int_{\xi}^{x}\left(c_{2}+\int_{\xi}^{w} 2 g(u) m(d u)\right) s(d w)
$$

for some constants $c_{1}, c_{2} \in \mathbb{R}$ and a function $g \in C_{0}$. In the case of boundary points in $I$, the constants $c_{1}, c_{2}$ have to satisfy the necessary boundary condition (2.2), or the respective boundary condition for the upper boundary point $b$, or both if both boundary points are in $I$. If $f \in \mathcal{D}$ is given as in (2.3), then it is immediate from (2.1) that $\mathcal{G} f=g$, and it can be shown that

$$
f\left(X_{t}\right)-\int_{0}^{t} \mathcal{G} f\left(X_{u}\right) d u, t \geq 0, \text { is a local martingale. }
$$

Now fix some measurable $h: I \rightarrow \mathbb{R}_{+}$satisfying for all $c<d$ in $I^{\circ}$ the condition

$$
\int_{[c, d]} h(x) m(d x)<\infty
$$

and define

$$
A_{t}=\int_{0}^{t} h\left(X_{u}\right) d u, \quad t \geq 0
$$


We write $H_{z}:=\inf \left\{t>0: X_{t}=z\right\}$. Note that $A$ may explode when $X$ approaches one of the boundary points of $I$. It is worth remarking that $A_{H_{a}-}<\infty=A_{H_{a}+}$ can happen.

The most common use of the first point of the next proposition is when $h \equiv 1$ and we characterise the exact conditions under which the boundary point is reached in finite time; see for example Rogers and Williams [21, Theorem V.51.2]. In the following result, we write $P^{x}$ for the law of $X$ when starting in $x \in I$.

Proposition 2.1 Suppose that the endpoint a is accessible, i.e., $s(a)>-\infty$. Then:

1) The following are equivalent:

(i) $\int_{a+}(s(x)-s(a)) h(x) m(d x)<\infty$;

(ii) For any $x_{0} \in I^{\circ}, P^{x_{0}}\left[A_{H_{a}-}<\infty\right]>0$.

2) The following are equivalent:

(i) $\int_{a+} h(x) m(d x)<\infty$;

(ii) $P^{a}\left[A_{H_{a}+}<\infty\right]=1$;

(iii) $P^{a}\left[A_{H_{a}+}<\infty\right]>0$.

Proof Writing $Y_{t}:=s\left(X_{t}\right)$, we have that $Y$ is a diffusion in natural scale with speed measure $m^{Y}$ defined by

$$
m^{Y}(s(x))=m(x)
$$

The additive functional $A$ is thus expressed equivalently as

$$
A_{t}=\int_{0}^{t}\left(h \circ s^{-1}\right)\left(Y_{u}\right) d u \text {. }
$$

Since $Y$ is in natural scale with speed measure $m^{Y}$, it can be represented as

$$
Y_{u}=B_{\tau_{u}}, \quad \tau_{u}^{Y}=\inf \left\{t: \Lambda_{t}^{Y}>u\right\}, \quad \Lambda_{t}^{Y}=\int_{s(I)} \ell(t, y) m^{Y}(d y),
$$

where $\ell$ is the local time of the Brownian motion $B$; see Rogers and Williams [21, Theorem V.74.1].

For 1), first assume that $0<\int_{[c, d]} h(u) m(d u)$ for all $c, d$ in $I^{\circ}$. Then we construct another diffusion $Z$ with speed measure $m^{Z}$ defined by

$$
m^{Z}(C):=\int_{C}\left(h \circ s^{-1}\right)(y) d m^{Y}(y) \quad \text { for all measurable } C \subseteq s(I)^{\circ},
$$

which is a regular diffusion by [21, Remark (ii) after (V.47.5)] and by our assumption (2.5) on $h$, and

$$
Z_{u}=B_{\tau_{u}^{Z}}, \quad \tau_{u}^{Z}=\inf \left\{t: \Lambda_{t}^{Z}>u\right\}, \quad \Lambda_{t}^{Z}=\int_{s(I)} \ell(t, y) m^{Z}(d y) .
$$

(We may suppose if we want that $B$ is the same Brownian motion as in the definition of $Y$, as we are only concerned here with distributional properties.) Without loss of 
generality, we may assume $s(a)=0$, and we write

$$
T_{0}=\inf \left\{t: B_{t}=0\right\}, \quad H_{0}^{Y}=\inf \left\{t: Y_{t}=0\right\}\left(=H_{a}^{X}\right), \quad H_{0}^{Z}=\inf \left\{t: Z_{t}=0\right\},
$$

and we note that $\tau_{H_{0}^{Y}}^{Y}=T_{0}=\tau_{H_{0}^{Z}}^{Z}$. The appropriate occupation time formula [21, Eq. (V.49.2)] also holds for positive measurable (instead of bounded measurable) functions, by monotone convergence. Therefore

$$
\int_{0}^{u} h\left(X_{s}\right) d s=\int_{s(I)} \ell\left(\tau_{u}^{Y}, y\right)\left(h \circ s^{-1}\right)(y) m^{Y}(d y)=\int_{s(I)} \ell\left(\tau_{u}^{Z}, z\right) m^{Z}(d z)=\Lambda_{\tau_{u}^{Z}}^{Z},
$$

so that $\Lambda_{t}^{Z}=\int_{0}^{\Lambda_{t}^{Y}} h\left(X_{s}\right) d s$ for all $t<T_{0}$ (where $\Lambda^{Y}$ and $\Lambda^{Z}$ are strictly increasing), and therefore

$$
A_{H_{a}^{X-}}=A_{H_{0}^{Y}-}=\int_{0}^{H_{0}^{Y}-} h\left(X_{s}\right) d s=H_{0}^{Z} .
$$

Statement 1) now follows immediately from [21, Theorem V.51.2] applied to the diffusion $Z$.

Now consider the general case where $\int_{[c, d]} h(u) m(d u)$ may vanish. We choose a positive function $f: I \rightarrow \mathbb{R}$ with $\int_{a+} f(u) m(d u)<\infty$. By the preceding argument, $P\left[\int_{0}^{H_{a}^{X}-} f\left(X_{s}\right) d s<\infty\right]>0$, so

$$
P\left[A_{H_{a}^{X}-}<\infty\right]>0 \Longleftrightarrow P\left[\int_{0}^{H_{a}^{X}-}(h+f)\left(X_{S}\right) d s<\infty\right]>0 .
$$

On the other hand, by our assumption on $f$,

$$
\begin{aligned}
& \int_{a+}(s(x)-s(a)) h(x) m(d x)<\infty \\
& \Longleftrightarrow \int_{a+}(s(x)-s(a))(h+f)(x) m(d x)<\infty .
\end{aligned}
$$

Thus 1) for the general case follows from the special case.

2) If we start $Y$ at the boundary point 0 and run until the first time $T_{1}^{Y}$ at which it reaches 1 , then $\tau_{T_{1}^{Y}}^{Y}=T_{1}:=\left\{t: B_{t}=1\right\}$ and some simple calculus gives

$$
\begin{aligned}
A_{T_{1}^{Y}} & =\int_{0}^{T_{1}^{Y}}\left(h \circ s^{-1}\right)\left(Y_{u}\right) d u \\
& =\int_{0}^{T_{1}}\left(h \circ s^{-1}\right)\left(B_{v}\right) d \Lambda_{v}^{Y} \\
& =\int_{s(I)}\left(h \circ s^{-1}\right)(y) \ell\left(T_{1}^{Y}, y\right) m^{Y}(d y) \\
& =\int_{I} h(x) \ell\left(T_{1}, s(x)\right) m(d x) .
\end{aligned}
$$


By the Ray-Knight theorem [21, Theorem VI.52.1], the process $y \mapsto \ell\left(T_{1}, 1-y\right)$ is a BESQ(2) diffusion started at 0 . So $E\left[\ell\left(T_{1}, 1-y\right)\right]$ is finite, continuous in $y$ and positive for $y>0$. Thus almost surely, $\ell\left(T_{1}, 1-y\right)$ is bounded for $y$ in $[0,1]$; hence if $\int_{a+} h(x) m(d x)<\infty$, it follows that $A_{H_{a}^{X}+}$ is a.s. finite - part (ii) of the statement and this implies part (iii) a fortiori. Going from part (iii) to part (i), if it were the case that $\int_{a+} h(x) m(d x)=\infty$, then since $\ell\left(T_{1}, s(x)\right)$ is a.s. bounded away from zero in a neighbourhood of 0 , it must be that $A_{H_{a}^{X}+}$ is a.s. infinite; a contradiction.

Remark 2.2 Of course, there is an analogous statement for an accessible upper boundary point.

Now suppose that $Z$ is a nonnegative continuous local martingale with $Z_{0}=1$. Provided $Z$ is a martingale, we can define a new probability $\tilde{P}$ on $\mathcal{F}_{\infty}$ by the recipe

$$
\left.\frac{d \tilde{P}}{d P}\right|_{\mathcal{F}_{t}}=Z_{t}, \quad t \geq 0,
$$

since by the martingale property, (2.6) implicitly defines a family of consistent finitedimensional distributions which can be extended using the Daniell-Kolmogoroff extension theorem; see Rogers and Williams [20, Theorem II.31.1]. To determine whether or not $Z$ is a martingale, define the stopping times

$$
T_{n}:=\inf \left\{t: Z_{t}>n\right\}, \quad n=2,3, \ldots,
$$

which reduce $Z$, and notice that it is possible to define for every positive integer $n$ a probability $\tilde{P}_{n}$ on $\mathcal{F}_{T_{n}}$ by

$$
\left.\frac{d \tilde{P}_{n}}{d P}\right|_{\mathcal{F}_{T_{n}}}=Z_{T_{n}} .
$$

But does the sequence $\left(\tilde{P}_{n}\right)_{n \in \mathbb{N}}$ extend to a probability measure $\tilde{P}$ on the whole of $\mathcal{F}$ ? The answer is in this simple result (see Stroock and Varadhan [23, Theorem 1.3.5]), whose proof we give for completeness.

Theorem 2.3 The local martingale $Z$ is a martingale if and only if for each $t>0$,

$$
\tilde{P}_{n}\left[T_{n} \leq t\right] \longrightarrow 0 \quad \text { as } n \rightarrow \infty
$$

Proof We have

$$
1=E\left[Z_{t \wedge T_{n}}\right]=E\left[Z_{t} 1_{\left\{t<T_{n}\right\}}\right]+E\left[Z_{T_{n}} 1_{\left\{T_{n} \leq t\right\}}\right]=E\left[Z_{t} 1_{\left\{t<T_{n}\right\}}\right]+\tilde{P}_{n}\left[T_{n} \leq t\right] .
$$

By monotone convergence, the first term on the right converges to $E\left[Z_{t}\right]$ so that (2.7) is equivalent to the statement that $E\left[Z_{t}\right]=1$ for all $t>0$, which is the condition that $Z$ is a martingale. 
Note that in the case where $Z$ is a martingale, we have $\tilde{P}_{n}=\tilde{P}$ on $\mathcal{F}_{T_{n}}$ for every $n$.

When is the condition (2.7) for $Z$ to be a martingale satisfied? To answer this, we define the 'reverse measure transformation'

$$
\tilde{Z}_{t}:=\frac{1}{Z_{t}}, \quad t \geq 0
$$

which is a positive $\tilde{P}$-martingale if $Z$ is a martingale. Obviously, we have

$$
T_{n}=\inf \left\{t: \tilde{Z}_{t}<n^{-1}\right\} .
$$

According to Theorem 2.3,

condition (2.7) $\Longleftrightarrow Z$ is a martingale $\Longleftrightarrow \tilde{P}$ is well defined.

The goal now is to determine when condition (2.7) is satisfied.

We need to be more specific about the local martingales $Z$ that we consider. If the diffusion $X$ was specified as the solution of an SDE

$$
d X_{t}=\sigma\left(X_{t}\right) d W_{t}+\beta\left(X_{t}\right) d t, \quad X_{0}=x_{0},
$$

with a pathwise unique strong solution and $C^{1}$-coefficients $\sigma>0$ and $\beta$, then we consider local martingales $Z$ of the form

$$
d Z_{t}=c\left(X_{t}\right) Z_{t} d W_{t}, \quad Z_{0}=1,
$$

where $c$ is assumed $C^{1}$ for convenience. The $\operatorname{SDE}$ (2.10) has the solution

$$
\begin{aligned}
Z_{t} & =\exp \left(\int_{0}^{t} c\left(X_{u}\right) d W_{u}-\frac{1}{2} \int_{0}^{t} c\left(X_{u}\right)^{2} d u\right) \\
& =\varphi\left(X_{t}\right) \exp \left(-\int_{0}^{t} \frac{\mathcal{G} \varphi}{\varphi}\left(X_{u}\right) d u\right),
\end{aligned}
$$

where $\mathcal{G}$ is the generator of $X$ and

$$
\log \varphi(x)=\int_{x_{0}}^{x} \frac{c}{\sigma}(y) d y .
$$

The equivalence of (2.11) and (2.12) is a simple exercise with Itô's formula, and is beside the point. The point is that the form (2.11) of $Z$ requires that the diffusion $X$ is specified as the solution of an SDE, but the form (2.12) does not. So we shall proceed to assume that $Z$ has the form (2.12) for some strictly positive function $\varphi \in \mathcal{D}$ which satisfies $\varphi\left(x_{0}\right)=1$. In this generality, it may happen that $\varphi$ vanishes in an endpoint of $I$, say e.g. $\varphi(a)=0$. In that case, the integral in (2.12) might diverge; but since $Z$ is a nonnegative local martingale and therefore a supermartingale, the limit $Z_{H_{a}-}:=\lim _{t \nearrow \nearrow H_{a}} Z_{t}$ exists almost surely and we may set $Z_{t}:=Z_{H_{a}-}$ while $X_{t}$ remains in $a$. 
The process $Z$ defined by (2.12) is still a local martingale, since using partial integration on (2.12) gives

$$
d Z_{t}=\left(d \varphi\left(X_{t}\right)-\mathcal{G} \varphi\left(X_{t}\right) d t\right) \exp \left(-\int_{0}^{t} \frac{\mathcal{G} \varphi}{\varphi}\left(X_{u}\right) d u\right),
$$

and $d \varphi\left(X_{t}\right)-\mathcal{G} \varphi\left(X_{t}\right) d t$ is the differential of a local martingale by (2.4).

The next question is how the change of measure (2.12) (if it is a change of measure) transforms the diffusion $X$. To answer this, we let $\tilde{\mathcal{D}}$ be the set of all functions $f$ such that $f \varphi \in \mathcal{D}$. Then using Itô's formula, it is a simple exercise to show that that for any $f \in \tilde{\mathcal{D}}$,

$$
Z_{t}\left(f\left(X_{t}\right)-\int_{0}^{t} \tilde{\mathcal{G}} f\left(X_{u}\right) d u\right), t \geq 0, \text { is a local martingale, }
$$

where

$$
\tilde{\mathcal{G}} f=\frac{1}{\varphi}(\mathcal{G}(f \varphi)-f \mathcal{G} \varphi) .
$$

The following result relates the form of $\tilde{\mathcal{G}}$ just found to the form (2.1).

Proposition 2.4 We have

$$
\tilde{\mathcal{G}}=\frac{1}{2} \frac{d^{2}}{d \tilde{m} d \tilde{s}}
$$

where $\tilde{s}$ and $\tilde{m}$ take the simple forms

$$
d \tilde{m}=\varphi^{2} d m, \quad d \tilde{s}=\varphi^{-2} d s .
$$

Proof Take some continuous finite-variation test function $\psi: I \rightarrow \mathbb{R}$ which vanishes off some compact set. In what follows, we assume that $I$ is open so that there are no boundary conditions to deal with, and we leave the checking of what happens in the other cases to the reader. Using integration by parts, we develop

$$
\begin{aligned}
& \int 2 \psi(x) \varphi(x) \tilde{\mathcal{G}} f(x) m(d x) \\
& =\int 2 \psi(\mathcal{G}(f \varphi)-f \mathcal{G} \varphi) d m=\int \psi\left(\frac{d}{d m}\left(\frac{d}{d s}(f \varphi)\right)-f \frac{d^{2}}{d m d s} \varphi\right) d m \\
& =-\int \frac{d}{d s}(f \varphi) d \psi+\int \frac{d \varphi}{d s} d(\psi f)=-\int \varphi \frac{d f}{d s} d \psi+\int \psi \frac{d \varphi}{d s} d f \\
& =-\int \varphi \frac{d f}{d s} d \psi+\int \psi \frac{d \varphi}{d s} \frac{d f}{d s} d s=-\int \varphi \frac{d f}{d s} d \psi+\int \psi \frac{d f}{d s} d \varphi \\
& =-\int d\left(\frac{\psi}{\varphi}\right) \varphi^{2} \frac{d f}{d s}=\int \frac{\psi}{\varphi} \frac{d}{d m}\left(\varphi^{2} \frac{d f}{d s}\right) d m=\int \psi \varphi \frac{d^{2} f}{d \tilde{m} d \tilde{s}} d m .
\end{aligned}
$$

Since $\psi$ is arbitrary, the result follows. 
One of the benefits of Proposition 2.4 is that there exists a measure $\hat{P}$ on the path space $C\left(\mathbb{R}_{+} ; I\right)$ such that $X$ is a one-dimensional diffusion with scale function $\tilde{s}$ and speed measure $\tilde{m}$; see Rogers and Williams [21, V.47]. Note that in the case where $Z$ is a martingale, $\hat{P}=\tilde{P}$, and that $\hat{P}=\tilde{P}_{n}$ on $\mathcal{F}_{T_{n}}$ for every $n$. So what we have to determine is

Question 1: Under $\hat{P}$, does $\tilde{Z}$ reach zero in finite time?

If not, then $Z$ is a martingale.

From now on, we make the simplifying assumption

Assumption 2.5 $\varphi$ has a continuous density with respect to $m$.

Since $\varphi \in \mathcal{D}$ by assumption, it is automatic that $\varphi$ has a continuous density with respect to $s$, but in general not with respect to $m$. Assumption 2.5 holds for example if both $s$ and $m$ have continuous densities with respect to Lebesgue measure, which is a situation covering many examples of interest.

Next, the $\hat{P}$-local martingale $\tilde{Z}$ can be expressed as

$$
\begin{aligned}
\tilde{Z}_{t} & =\exp \left(-\log \varphi\left(X_{t}\right)-\int_{0}^{t} \varphi \tilde{\mathcal{G}}(1 / \varphi)\left(X_{u}\right) d u\right) \\
& =\exp \left(\tilde{M}_{t}-\frac{1}{2}\langle\tilde{M}\rangle_{t}\right)
\end{aligned}
$$

for some continuous $\hat{P}$-local martingale $\tilde{M}$, where the representation (2.16) follows from the equality $\varphi^{-1} \mathcal{G} \varphi=-\varphi \tilde{\mathcal{G}}(1 / \varphi)$, an immediate consequence of (2.14). If we make the Itô expansion of $\log \tilde{Z}$ given in (2.16), we find after some calculations and simplifications that the finite-variation part of $\log \tilde{Z}$ is

$$
-\frac{1}{2} \tilde{h}\left(X_{t}\right) d t
$$

where

$$
\tilde{h}=\frac{d \varphi}{d \tilde{m}} \frac{d \varphi}{d s}=\frac{1}{\varphi^{2}} \frac{d \varphi}{d \tilde{m}} \frac{d \varphi}{d \tilde{s}}=\frac{1}{\varphi^{2}} \frac{d \varphi}{d m} \frac{d \varphi}{d s} .
$$

Hence by comparing (2.17) and (2.18), we learn that

$$
d\langle\tilde{M}\rangle_{t}=\tilde{h}\left(X_{t}\right) d t
$$

In particular, $\tilde{h}$ is nonnegative. So under Assumption 2.5, it is now clear that to answer Question 1, we have to answer:

Question 2: Under $\hat{P}$, does

$$
\tilde{A}_{t}:=\int_{0}^{t} \tilde{h}\left(X_{u}\right) d u, \quad t \geq 0,
$$

reach infinity in finite time? 
Remark 2.6 When the diffusion is specified as the solution to an SDE, (2.20) appears at Mijatović and Urusov [17, Eq. (9)], [16, Eq. (2.6)]. If $X$ is the solution to an SDE, then

$$
\begin{aligned}
s^{\prime}(x) & =\exp \left(-2 \int_{x_{0}}^{x} \frac{\beta(u)}{\sigma(u)^{2}} d u\right), \\
m^{\prime}(x) & =\frac{1}{\sigma(x)^{2} s^{\prime}(x)},
\end{aligned}
$$

so

$$
\tilde{h}=\frac{1}{\varphi^{2}} \frac{d \varphi}{d m} \frac{d \varphi}{d s}=\left(\frac{\varphi^{\prime}}{\varphi}\right)^{2} \frac{1}{m^{\prime} s^{\prime}}=\left(\frac{\varphi^{\prime}}{\varphi}\right)^{2} \sigma^{2}=\left(\log (\varphi)^{\prime}\right)^{2} \sigma^{2}=\left(\frac{c}{\sigma}\right)^{2} \sigma^{2}=c^{2} .
$$

Now we address Question 2. If $K \subseteq I^{\circ}$ is any compact set and if we define $\zeta=\inf \left\{t: \tilde{A}_{t}=\infty\right\}<\infty$, then clearly $X$ must exit $K$ before $\zeta$, because the integrand in (2.20) is bounded on $K$ by Assumption 2.5. By considering an increasing sequence of compact sets $K_{n}$ increasing to $I^{\circ}$, we see that if $\tilde{A}$ reaches infinity in finite time, it has to be at a time when $X$ reaches a boundary point of $I$.

To understand this, we look at the diffusion $Y=\tilde{s}(X)$ which is a diffusion in natural scale under $\hat{P}$, taking values in the interval $\tilde{s}(I)$ whose endpoints are $\tilde{a}:=\tilde{s}(a)<\tilde{b}:=\tilde{s}(b)$. Two cases arise.

CASE 1: $\tilde{a}$ and $\tilde{b}$ are both infinite. Since $Y$ is a continuous local martingale under $P$ and therefore a time-change of Brownian motion, see e.g. Revuz and Yor [19, Theorem V.1.7], $Y$ cannot reach either endpoint in finite time; so the change-of-measure local martingale $Z$ is a true martingale under $P$.

CASE 2: One at least of $\tilde{a}$ and $\tilde{b}$ is finite. To fix ideas, let us suppose that $\tilde{a}$ is finite and $\tilde{b}=\infty$, and see what happens at $\tilde{a}$; the treatment at a finite upper boundary point is analogous.

First, we have to ask whether $Y$ reaches the lower boundary point $\tilde{a}$ in finite time. According to Proposition 2.1 (with $h \equiv 1$, cf. Remark 2.2 (ii)), this happens if and only if

$$
\int_{a+}(\tilde{s}(x)-\tilde{s}(a)) \tilde{m}(d x)<\infty .
$$

If $Y$ does not reach $\tilde{a}$ in finite time, then explosion of $\tilde{A}$ in finite time is clearly impossible.

However, if $Y$ does reach $\tilde{a}$ in finite time, then the additive functional $\tilde{A}$ may explode at that time. In the situation considered by Mijatović and Urusov [17] where the diffusion $X$ stops at $a$ if it ever gets there, the criterion for explosion is (according to Proposition 2.1)

$$
\int_{a+}(\tilde{s}(x)-\tilde{s}(a)) \tilde{h}(x) \tilde{m}(d x)=\infty .
$$

On the other hand, if the diffusion $Y$ reflects off $\tilde{a}$, then explosion could happen at $H_{a}+$ even though there was no explosion at $H_{a}-$, and the criterion for $\tilde{A}$ to explode 
at $H_{a}+$ is now

$$
\int_{a+} \tilde{h}(x) \tilde{m}(d x)=\infty
$$

by Proposition 2.1, part 2). For the applications of interest to us, this is the relevant criterion, as the CIR diffusions we deal with later all reflect off the boundary point.

Notice that condition (2.23) can be equivalently expressed (due to the form (2.19) of $\tilde{h}$ and (2.15)) as

$$
\int_{a+} \frac{d \varphi}{d s}(x) \frac{d \varphi}{d m}(x) m(d x)=\infty .
$$

Thus we see that in order to decide whether the local $P$-martingale $Z$ is not a true $P$-martingale, we have to answer the following three questions (under $\hat{P}$ ):

1) Is at least one of the endpoints $\tilde{a}, \tilde{b}$ of $\tilde{s}(I)$ finite?

2) If $\tilde{a}$ (say) is finite, does $X$ reach $a$ in finite time (see (2.21))?

3 ) If so, does $\tilde{A}$ explode when $X$ reaches $a$ (see (2.23))?

To summarise then, we have the following result.

Theorem 2.7 Let $X$ be a diffusion on the interval $I \subseteq \mathbb{R}, X_{0}=x_{0} \in I, a:=\inf I$, $b:=\sup I$, with scale function $s$ and speed measure $m$. We define the change-ofmeasure local martingale $Z$ by

$$
Z_{t}=\varphi\left(X_{t}\right) \exp \left(-\int_{0}^{t} \frac{\mathcal{G} \varphi}{\varphi}\left(X_{u}\right) d u\right), \quad t \geq 0,
$$

where $\mathcal{G}$ is the generator of $X$ and $\varphi$ is strictly positive and $C^{1}$ with $\varphi\left(x_{0}\right)=1$. Let $\hat{P}$ be a probability measure on $C([0, \infty))$ under which $X$ is a diffusion with scale function $\tilde{s}$ and speed measure $\tilde{m}$, where

$$
d \tilde{m}=\varphi^{2} d m \quad \text { and } \quad d \tilde{s}=\varphi^{-2} d s .
$$

Denote $\tilde{a}=\tilde{s}(a), \tilde{b}=\tilde{s}(b)$. Assume further Assumption 2.5. Suppose that all of the following three conditions are satisfied:

1) At least one of the endpoints $\tilde{a}, \tilde{b}$ is finite.

2) At least one of the finite endpoints is reached in finite time under $\hat{P}$ (see (2.21)).

3) There is a finite endpoint which is reached in finite time and at which the additive functional $\tilde{A}$ explodes under $\hat{P}$ (see (2.23) or (2.22)).

Then the change-of-measure local P-martingale $Z$ is not a true P-martingale. Otherwise, it is.

Remark 2.8 In Çetin [3], a similar question to ours is discussed, by giving a class of absolutely continuous measure changes using potentials. More specifically, we characterise the positive functions $\varphi$ for which $Z$ is a true martingale, whereas [3, Theorem 3.2] shows that if $\varphi$ is a potential, then $\mathrm{Z}$ is a true martingale. The key differences are that [3] works in an SDE setting and that the statement of [3, Theorem 3.2] 
assumes $X$ has a semimartingale local time; but the most general one-dimensional diffusion such as we work with need not be a semimartingale.

If $Z$ is a martingale under $P$, then the recipe (2.6) defines a new measure $\tilde{P}$ on the path space under which the canonical process is again a regular diffusion. The law $\tilde{P}$ is therefore absolutely continuous with respect to $P$, but not in general equivalent. For checking whether $Z$ actually is a martingale, we use the measure $\hat{P}$ and Theorem 2.7. Here are two interesting examples, where the process is given by the SDE recipe (2.9) and the change-of-measure local martingale is of the form (2.10).

Example 2.9 A canonical example is when $X$ solves (2.9) with $\sigma(x) \equiv 1, \beta(x) \equiv 0$, on $I=[0, \infty)$ with $X_{0}=x_{0}>0$. That is, $X$ is of the form $X_{t}=x+W_{t \wedge H_{0}}$, where $W$ is a standard Brownian motion and $H_{0}$ is the time when $X$ hits $\{0\}$. We want $c(x)=1 / x$ so that $X$ solves the $\operatorname{BES}(3) \mathrm{SDE}$

$$
d X_{t}=\frac{1}{X_{t}} d t+d \tilde{W}_{t}, \quad X_{0}=x_{0},
$$

under $\hat{P}$. In this example, $a=0, b=\infty, s(x)=x$ and $m^{\prime}(x)=1$. From (2.13) and (2.15), we find that $\varphi(x)=x / x_{0}$, and we may take $\tilde{s}(x)=-x_{0}^{2} / x, \tilde{m}^{\prime}(x)=x^{2} / x_{0}^{2}$, and therefore $\tilde{a}=-\infty, \tilde{b}=0$. According to our method, we next ask whether the finite boundary point $\tilde{b}$ can be reached in finite time. By the integral test (2.21) (in the analogous form for an upper boundary), the process $X$ approaches $\infty$ (or $\tilde{s}(X)$ approaches 0 ) under $\hat{P}$, but never gets there.

Thus by Theorem 2.7, there is an absolutely continuous change of measure, taking Wiener measure $P$ to the law $\hat{P}$ of $\operatorname{BES}(3)$ started at $x_{0}$, which is absolutely continuous with respect to Wiener measure $P$. Here $\hat{P}$ is not equivalent to $P$, since $Z \equiv X$ is not a.s. positive.

Note that in this example, we knew from the outset that $Z$ is a true martingale, but nevertheless, the application of our recipe is illuminating.

Example 2.10 An important example for the CIR process (1.2) followed by the volatility in the Heston model is the case where under $P$, the diffusion $X$ follows

$$
d X_{t}=2 \sqrt{X_{t}^{+}} d W_{t}+\delta_{0} d t, \quad X_{0}=x_{0}>0,
$$

the squared-Bessel SDE of dimension $\delta_{0}>0$. See Revuz and Yor [19, Chap. XI] for a definitive account. Suppose that we want to perform a measure change to transform the SDE to

$$
d X_{t}=2 \sqrt{X_{t}^{+}} d \tilde{W}_{t}+\delta_{1} d t, \quad X_{0}=x_{0}>0,
$$

where again $\delta_{1}>0$. This requires us to add a drift $c\left(X_{t}\right) d t$ to $d W_{t}$ in (2.24), where

$$
c(x)=\left(\delta_{1}-\delta_{0}\right) /(2 \sqrt{x}) .
$$

Simple calculations give

$$
\varphi(x)=x^{\left(\delta_{1}-\delta_{0}\right) / 4},
$$


taking $x_{0}=1$ with no real loss of generality. The scale function $\tilde{s}$ is given by

$$
\tilde{s}^{\prime}(x)=\exp \left(-\int^{x} \frac{2 \delta_{1}}{4 y} d y\right)=\exp \left(-\frac{1}{2} \delta_{1} \log x\right)=x^{-\delta_{1} / 2},
$$

so that (up to irrelevant constants)

$$
\tilde{s}(x)= \begin{cases}x^{\left(2-\delta_{1}\right) / 2}, & \text { if } \delta_{1} \neq 2, \\ \log x, & \text { if } \delta_{1}=2 .\end{cases}
$$

There are three cases to understand:

1) $0<\delta_{1}<2$ : Here, $\tilde{a}=0$ and $\tilde{b}=\infty$. The criterion (2.21) shows that $\tilde{a}$ is reached in finite time under $\hat{P}$, and the criterion (2.23) requires us to calculate

$$
\int_{a+} \tilde{h}(x) \tilde{m}(d x)=\int_{0+}\left(\frac{\varphi^{\prime}(x)}{\varphi(x)}\right)^{2} \frac{1}{\tilde{s}^{\prime}(x)} d x=\frac{\left(\delta_{0}-\delta_{1}\right)^{2}}{16} \int_{0+} x^{-2+\delta_{1} / 2} d x=\infty .
$$

So in this case, there is never an absolutely continuous change of measure which achieves the desired drift, whatever $\delta_{0} \neq \delta_{1}$.

2) $\delta_{1}=2$ : In this case, $\tilde{s}(x)=\log x$, thus $\tilde{a}=-\infty$ and $\tilde{b}=\infty$. So the first check of our recipe fails, and there is an absolutely continuous measure change that achieves the desired drift.

3) $\delta_{1}>2$ : This time, $\tilde{s}(x)=-x^{-\left(\delta_{1}-2\right) / 2}$, so $\tilde{b}=0, \tilde{a}=-\infty$. However, the criterion (2.21) is infinite for approaching $\tilde{b}$, so $X$ approaches but never reaches $\infty$ under $\hat{P}$, and there is an absolutely continuous measure change which turns the dynamics of $X$ into (2.25).

So to summarise, if we want to use a change of measure to change the dimension of a $\operatorname{BESQ}\left(\delta_{0}\right)$ to $\delta_{1} \neq \delta_{0}$, this is

- never possible if $\delta_{1}<2$,

- always possible if $\delta_{1} \geq 2$.

It may be surprising that this dichotomy is not affected by the value of $\delta_{0}$. Here is some intuition about the result. Informally, the change-of-measure local martingale is a true martingale if when we see a path of the diffusion under $\hat{P}$, we can never be certain that we are not looking at a path of the diffusion under $P$. If $\delta_{1} \geq 2$, then the paths under $\hat{P}$ will never reach 0 , and this is the only place where we can expect to see immediately whether the law is $P$ or $\hat{P}$. On the other hand, if $\delta_{1}<2$, then the path will eventually reach 0 , and the fine structure of the path at $H_{0}+$ will reveal which value of $\delta$ is the truth.

\section{Arbitrage opportunities in the Heston model}

As is well known, the SDE (1.2) for the Heston volatility has a pathwise unique strong solution from any nonnegative starting point. The following fact about the strict positivity of a CIR process is also well known; see for example Göing-Jaeschke and Yor [9]. 
Lemma 3.1 For the CIR process $v$ specified by (1.2), the following are equivalent:

(i) $P\left[\forall t \in(0, T]: v_{t}>0\right]=1$.

(ii) $2 \kappa \theta \geq \sigma^{2}$ (Feller condition).

By scaling time in the CIR SDE (1.2) to convert the volatility $\sigma$ to the canonical value 2 appearing in the BESQ SDE (2.24), we see that the Feller condition is equivalent to the statement that the effective dimension of the CIR process is at least 2 ,

$$
\delta:=\frac{4 \kappa \theta}{\sigma^{2}} \geq 2
$$

Definition 3.2 A probability measure $\tilde{P}$ on $\mathcal{F}$ is an equivalent local martingale measure (ELMM) if

(i) for all $A \in \mathcal{F}$, one has $\tilde{P}[A]=0$ iff $P[A]=0$;

(ii) the process $S$ is a local martingale under $\tilde{P}$.

The following result is a direct consequence of standard results about ELMMs, which can be found for example in Williams [24, Lemma 5.4.2 and Theorem 5.4.3].

Lemma 3.3 Let $\tilde{P}$ be an ELMM for the generalised Heston model (1.1), (1.2). Then there exist predictable processes $\gamma, \gamma^{\prime}$, both locally square-integrable, such that

(i) the process $Z$ with $Z_{t}:=e^{M_{t}-\frac{1}{2}[M]_{t}}$ with

$$
M_{t}:=\int_{0}^{t} \gamma_{t} d W_{t}+\int_{0}^{t} \gamma_{t}^{\prime} d W_{t}^{\prime}, \quad t \in[0, T],
$$

is a martingale;

(ii) $Z_{T}$ is a density for $\tilde{P}$;

(iii) the integrand $\gamma$ satisfies

$$
\mu\left(v_{t}\right)+\sqrt{v_{t}}\left(\rho \gamma_{t}+\rho^{\prime} \gamma_{t}^{\prime}\right)=0 \quad \text { for a.e. } t \in[0, T]
$$

(iv) $\left(S_{t}\right)_{t \in[0, T]}$ is a local martingale with respect to $\tilde{P}$ iff $\left(Z_{t} S_{t}\right)_{t \in[0, T]}$ is a local martingale with respect to $P$.

Theorem 3.4 Suppose that the Feller condition (3.1) fails, i.e., $\delta<2$. Then:

1) The generalised Heston model (1.1), (1.2) admits no ELMM if $\mu(0) \neq 0$.

2) The generalised Heston model (1.1), (1.2) has an ELMM if

$$
\int_{0+} \mu(x)^{2} x^{-2+\delta / 2} d x<\infty .
$$

Proof 1) Assume by way of contradiction that there exists an ELMM. By Lemma 3.3, there exists a martingale $Z$ such that $\left(S_{t} Z_{t}\right)_{t \in[0, T]}$ is a $P$-local martingale and

$$
d Z_{t} / Z_{t}=\gamma_{t} d W_{t}+\gamma_{t}^{\prime} d W_{t}^{\prime}, \quad t \in[0, T],
$$


with predictable locally square-integrable, and therefore a.s. pathwise square-integrable, processes $\gamma, \gamma^{\prime}$ satisfying (3.2). Using the continuity of $\mu, \mu(0) \neq 0$ and the fact that $\gamma, \gamma^{\prime}$ are a.s. square-integrable, this implies

$$
\int_{0}^{T} \frac{1}{v_{t}} d t<\infty \quad P \text {-a.s. }
$$

By Lemma 3.1, $P\left[\forall t \in[0, T]: v_{t}>0\right]<1$. Therefore, if we define

$$
\tau_{0}:=\inf \{t \geq 0: v(t)=0\} \wedge T,
$$

we have $P\left[\tau_{0}<T\right]>0$ and in particular

$$
P\left[v_{\tau_{0}}=0\right]>0 .
$$

On the other hand, by Itô's formula,

$$
\log v_{\tau_{0}}=\log v_{0}+\int_{0}^{\tau_{0}} \frac{\sigma}{\sqrt{v_{t}}} d W_{t}+\int_{0}^{\tau_{0}}\left(\frac{2 \kappa \theta-\sigma^{2}}{2 v_{t}}-\kappa\right) d t
$$

From (3.4), we get

$$
\int_{0}^{\tau_{0}} \frac{1}{v_{t}} d t<\infty \quad P \text {-a.s. }
$$

so that both integrals in (3.6) are finite a.s., and therefore we obtain that

$$
P\left[\log v_{\tau_{0}}>-\infty\right]=P\left[v_{\tau_{0}}>0\right]=1 .
$$

But this contradicts (3.5).

2) It is to be expected that if there is an ELMM, there will be many; so to prove the second statement, we shall identify one. We choose to take the change-of-measure martingale to be

$$
\frac{d Z_{t}}{Z_{t}}=-\frac{\mu\left(v_{t}\right)}{\rho^{\prime} \sqrt{v_{t}}} d W_{t}^{\prime}=: c\left(v_{t}\right) d W_{t}^{\prime}, \quad Z_{0}=1 .
$$

We see that provided $Z$ is a martingale, the drift of $S$ becomes 0 and the dynamics of $v$ is unchanged. So we need to show that $Z$ is a true martingale, and for this we use Theorem 2.3 and the arguments of Sect. 2. As before at (2.8), we define

$$
\tilde{Z}_{t}:=\frac{1}{Z_{t}}=\exp \left(\int_{0}^{t}-c\left(v_{s}\right) d \tilde{W}^{\prime}{ }_{s}-\frac{1}{2} \int_{0}^{t} c\left(v_{s}\right)^{2} d s\right) .
$$

Here, $d \tilde{W}_{t}^{\prime}=d W_{t}^{\prime}+c\left(v_{t}\right) d t$. Noticing that $\tilde{Z}$ can be written as

$$
\tilde{Z}_{t}=\exp \left(B_{A_{t}}-A_{t}\right)
$$

for some Brownian motion $B$, with $A_{t}:=\int_{0}^{t} c\left(v_{s}\right)^{2} d s$, it is clear that Question 1 from Sect. 2 is now equivalent to 
Question $2^{\prime}$ : Under $\hat{P}$, does $A_{t}:=\int_{0}^{t} c\left(v_{s}\right)^{2} d s$ reach infinity in finite time?

This is a question about the CIR process $v$. The scale function of $v$ is given by

$$
\tilde{s}^{\prime}(v)=\exp \left(-2 \int_{0}^{v} \frac{\kappa(\theta-x)}{\sigma^{2} x} d x\right)=v^{-\delta / 2} e^{2 \kappa v / \sigma^{2}} .
$$

The scale function $\tilde{s}$ is therefore finite at 0 , since $\delta<2$, and $v$ will reach 0 in finite time under $\hat{P}$. The criterion that $A$ does not explode under $\hat{P}$ is (see Proposition 2.1) that

$$
\int_{0+} c(x)^{2} \tilde{m}(d x)=\int_{0+} c(x)^{2} \frac{d x}{\sigma(x)^{2} \tilde{s}^{\prime}(x)} \asymp \int_{0+} \mu(x)^{2} x^{-2+\delta / 2} d x
$$

should be finite, and this is condition (3.3). The symbol $\asymp$ in (3.8) means that the ratio of the two sides is bounded and bounded away from 0 .

Remark 3.5 Similar calculations as those in the preceding proof of the first statement appear in Guo [10], where it is shown that there is no ELMM if the stock price process itself is a CIR process and the Feller condition does not hold.

Corollary 3.6 The classical Heston model with constant drift $\mu \neq 0$ does not admit an ELMM if the Feller condition is not satisfied.

The significance of this result lies in the fact that by the famous fundamental theorem of asset pricing (FTAP), the non-existence of an ELMM implies the existence of a free lunch with vanishing risk, i.e., a weak form of arbitrage; see Delbaen and Schachermayer [5, 6]. We give an explicit construction in the Appendix.

Finally, for completeness, we record the following little result which tells us what happens when the Feller condition holds.

Theorem 3.7 Suppose that the Feller condition (3.1) holds, i.e., $\delta \geq 2$. Then there is always an ELMM.

Proof Recall our standing assumption that $\mu$ is continuous. We use the same changeof-measure martingale (3.7) as for the proof of Theorem 3.4, part 2). Exactly as there, we need to establish that $A_{t}:=\int_{0}^{t} c\left(v_{s}\right)^{2} d s$ remains finite for all time. But we have

$$
c(v):=-\frac{\mu(v)}{\rho^{\prime} \sqrt{v}}
$$

and since the CIR process remains strictly positive for all $t>0$ by Lemma 3.1 and does not explode, it follows immediately that if $v_{0}>0$, then $A$ does not explode. If $v_{0}=0$, a separate argument is required, which we leave to the reader. 


\section{Conclusion}

We have provided a complete characterisation of when the change-of-measure local martingale that transforms a one-dimensional diffusion to another one with a different drift is a true martingale. We are able to decide this question by a simple three-step algorithm (compare Theorem 2.7). This has practical implications for a generalised Heston model that allows a volatility-dependent growth rate: We can show the absence of arbitrage if a simple integrability condition holds, even when the Feller condition is violated. This extends the results for the classical Heston model with constant growth rate different from the riskless rate, for which we have shown that no ELMM exists and thus arbitrage opportunities are incurred in that case.

Acknowledgements Sincere thanks are due to Ralf Korn for many fruitful discussions in the early stages of this project. We also appreciate helpful comments of David Criens and an anonymous referee. Moreover, we wish to thank Martin Schweizer for a very careful reading of this manuscript.

S. Desmettre und G. Leobacher are supported by the Austrian Science Fund (FWF) projects F5507N26 and F5508-N26, which are part of the Special Research Program Quasi-Monte Carlo Methods: Theory and Applications. S. Desmettre moreover appreciates support by the DFG Research Training Group 1932 Stochastic Models for Innovations in the Engineering Sciences.

Funding Note Open access funding provided by Johannes Kepler University Linz.

Publisher's Note Springer Nature remains neutral with regard to jurisdictional claims in published maps and institutional affiliations.

Open Access This article is licensed under a Creative Commons Attribution 4.0 International License, which permits use, sharing, adaptation, distribution and reproduction in any medium or format, as long as you give appropriate credit to the original author(s) and the source, provide a link to the Creative Commons licence, and indicate if changes were made. The images or other third party material in this article are included in the article's Creative Commons licence, unless indicated otherwise in a credit line to the material. If material is not included in the article's Creative Commons licence and your intended use is not permitted by statutory regulation or exceeds the permitted use, you will need to obtain permission directly from the copyright holder. To view a copy of this licence, visit http://creativecommons.org/licenses/by/ $4.0 \%$.

\section{Appendix: Making an FLVR in the Heston model}

The main result of Delbaen and Schachermayer [5] is that for a locally bounded semimartingale, the existence of an equivalent local martingale measure is a condition equivalent to the absence of a free lunch with vanishing risk. The following lemma follows readily from the definition of free lunch with vanishing risk (FLVR); see [5, Definition 2.8].

Lemma A.1 Suppose that there exists a sequence $f_{n}:=\left(H_{n} \cdot S\right)_{\infty}$ of admissible terminal wealths with the following properties:

1) The negative parts $f_{n}^{-}$tend uniformly to zero.

2) The $f_{n}$ tend almost surely to some nonnegative limit $f_{\infty}$ which is not almost surely zero.

Then there exists an FLVR. 
Proof We need to construct a sequence $\left(K_{m}\right)$ of admissible strategies, a sequence $\left(g_{m}\right)$ of bounded measurable functions with $\left(K_{m} \cdot S\right)_{\infty} \geq g_{m}$ and a measurable function $g_{\infty}$ which is nonnegative and positive with positive probability such that $\lim _{m \rightarrow \infty}\left\|g_{m}-g_{\infty}\right\|_{\infty}=0$.

Without loss of generality, we may assume that $f_{n} \geq-n^{-1}$ for all $n$, by passing to a subsequence if necessary. We have $f_{n} \wedge 1 \rightarrow f_{\infty} \wedge 1$ a.s. Note that we also have $-1 \leq-n^{-1} \leq f_{n} \wedge 1 \leq 1$ and $0 \leq f_{\infty} \wedge 1 \leq 1$. Let $A:=\left\{f_{\infty} \wedge 1>0\right\}$. By assumption, $P[A]>0$. By Egorov's theorem, there exist a measurable set $B \subseteq A$ with $P[B]>0$ and a subsequence $\left(n_{m}\right)$ such that uniformly on $B$,

$$
f_{n_{m}} \wedge 1 \longrightarrow f_{\infty} \wedge 1 \quad \text { as } m \rightarrow \infty
$$

We now define $K_{m}:=H_{n_{m}}, g_{m}:=\left(f_{n_{m}} \wedge 1\right) 1_{B}-n_{m}^{-1} 1_{\Omega \backslash B}$ and $g_{\infty}:=\left(f_{\infty} \wedge 1\right) 1_{B}$, which have the required properties.

We now directly construct a sequence $\left(f_{n}\right)$ with the above properties and thus an FLVR. Then it follows from Delbaen and Schachermayer [5] that there is no equivalent local martingale measure. We concentrate on the finite-horizon case. This is not a limitation: if we can construct an FLVR over $[0, T]$ with $0<T<\infty$, then we get an FLVR over $[0, \infty)$ by simply investing our gains up to time $T$ in the riskless asset after time $T$.

To fix ideas, we assume with no real loss of generality that $r=0$ and $\mu>0$; if $\mu=0$, then we are already under an equivalent local martingale measure and there is nothing interesting to say, and if $\mu<0$, the argument we give carries through by reversing signs in the appropriate places.

We first reduce the problem to a simpler canonical form, by modifying the SDE for $v$ to

$$
d v_{t}=\sigma \sqrt{v_{t}} d W_{t}+\kappa \theta d t
$$

We can always do this because if we can construct an FLVR in this setting, we can perform an absolutely continuous change of measure to change the drift in (A.1) into the original drift in (1.2), and null events (and therefore an FLVR) will not be changed by this (of course, this will change the drift in (1.1), but an equivalent change of measure to $W^{\prime}$ can be applied to reverse this). Once we have done this, we have that $v$ is a BESQ process, or at least a BESQ process run at a constant speed which need not be 1 . Again, we change nothing that matters by rescaling the speed so that we are looking at an actual BESQ process, i.e.,

$$
d v_{t}=2 \sqrt{v_{t}} d W_{t}+\delta d t
$$

where we have the correspondence $\delta=4 \kappa \theta / \sigma^{2}$. Thus the Feller condition (3.1) is the statement that $\delta<2$, the familiar condition in terms of the dimension $\delta$ of the BESQ process that the process hits 0 . For more background on BESQ processes, we refer to Revuz and Yor [19, Chap. XI, §1].

Looking at (1.1), it is rather obvious what the idea of the construction should be: we need to go long in the asset when $v$ is very small because at such times, the 
positive drift $\mu$ will dominate the tiny variance. Ideally, we could just hold the asset at the times when $v$ is equal to zero, because then the martingale part of the gainsfrom-trade process would vanish and we should just get the drift contribution; but this does not work because the Lebesgue measure of the set of times when $v=0$ is zero, see for example [19, Proposition 1.5]. So the next attempt is to hold the asset only at times when $v_{t}<\varepsilon$ for some very small $\varepsilon>0$, which we hope will be an approximate arbitrage. As we shall see, this leads us to an FLVR.

In order to do this, we must be able to do some calculations on BESQ processes, which turn out to be easier in terms of the scale and speed representation of $v$ in terms of a standard Brownian motion. A scale function of $v$ is easily verified to be

$$
s(x)=x^{1-\delta / 2} .
$$

If we then consider the diffusion in natural scale, $Y_{t}=s\left(v_{t}\right)=v_{t}^{1-\delta / 2}$, and apply Itô's formula (as the scale function is not $C^{2}$, this is only valid in the region $(0, \infty)$ where $s$ is $C^{2}$ ), we find that

$$
d Y_{t}=(2-\delta) v_{t}^{(1-\delta) / 2} d W_{t}=(2-\delta) Y_{t}^{(1-\delta) /(2-\delta)} d W_{t},
$$

at least while $Y$ is strictly positive. Clearly (A.3) cannot hold for all time, because otherwise $Y$ would be a nonnegative local martingale and would have to stick at 0 once it reaches 0 . Of course, this does not happen, and this is because of a local-time effect at zero; see Rogers and Williams [21, V.48.6] for more details. But (A.3) tells us that away from 0 , the speed measure for $Y$ is

$$
m(d y)=\frac{d y}{(2-\delta)^{2}}|y|^{2(\delta-1) /(2-\delta)},
$$

and the speed measure does not charge 0 because $Y$ spends no time there. So we may create a weak solution to (A.2) starting from a standard Brownian motion $B$ with local time process $\{\ell(t, x): x \in \mathbb{R}, t \geq 0\}$ by the recipe

$$
\begin{aligned}
\Lambda_{t} & :=\int \ell(t, a) m(d a)=\int_{0}^{t} \frac{\left|B_{u}\right|^{2(\delta-1) /(2-\delta)}}{(2-\delta)^{2}} d u, \\
\tau_{t} & =\inf \left\{u: \Lambda_{u}>t\right\}, \\
Y_{t} & =\left|B_{\tau_{t}}\right|, \\
v_{t} & =Y_{t}^{2 /(2-\delta)}
\end{aligned}
$$

see [21, V.47, V.48] for more details.

The idea now is to make a portfolio $\varphi\left(Y_{t}\right) / S_{t}$ so that the gains-from-trade process becomes

$$
G_{t}:=\int_{0}^{t} \varphi\left(Y_{u}\right)\left(\sqrt{v_{u}} d \hat{W}_{u}+\mu d u\right),
$$

where $d \hat{W}=\rho d W+\rho^{\prime} d W^{\prime}$; see (1.1). We do this in such a way that the local martingale term in (A.4) is negligible, and the Lebesgue term is not. To explain, when we 
look at the Lebesgue integral in $G_{t}$, we see $\mu$ times

$$
\begin{aligned}
\int_{0}^{t} \varphi\left(Y_{u}\right) d u & =\int_{0}^{t} \varphi\left(\left|B_{\tau_{s}}\right|\right) d s=\int_{0}^{\tau_{t}} \varphi\left(\left|B_{u}\right|\right) d \Lambda_{u} \\
& =\int_{0}^{\tau_{t}} \varphi\left(\left|B_{u}\right|\right) \frac{\left|B_{u}\right|^{2(\delta-1) /(2-\delta)}}{(2-\delta)^{2}} d u .
\end{aligned}
$$

If we now choose $\varepsilon>0$ and define

$$
\varphi(x)=(2 \varepsilon)^{-1} I_{[0, \varepsilon]}(x) x^{2(1-\delta) /(2-\delta)}(2-\delta)^{2},
$$

we find that as $\varepsilon \downarrow 0$,

$$
\int_{0}^{t} \varphi\left(Y_{u}\right) d u=(2 \varepsilon)^{-1} \int_{0}^{\tau_{t}} I_{[0, \varepsilon]}\left(\left|B_{u}\right|\right) d u=\frac{1}{2 \varepsilon} \int_{-\varepsilon}^{\varepsilon} \ell\left(\tau_{t}, x\right) d x \longrightarrow \ell\left(\tau_{t}, 0\right) .
$$

The quadratic variation of the martingale part of $G$ is

$$
\begin{aligned}
\langle G\rangle_{t} & =\int_{0}^{t} \varphi\left(Y_{u}\right)^{2} v_{u} d u=\int_{0}^{t} \varphi\left(Y_{u}\right)^{2} Y_{u}^{2 /(2-\delta)} d u \\
& =\int_{0}^{\tau_{t}} \varphi\left(\left|B_{u}\right|\right)^{2}\left|B_{u}\right|^{2 /(2-\delta)} d \Lambda_{u} \\
& =\frac{(2-\delta)^{2}}{4 \varepsilon^{2}} \int_{0}^{\tau_{t}} I_{[0, \varepsilon]}\left(\left|B_{u}\right|\right)\left|B_{u}\right|^{2} d u \\
& =\frac{(2-\delta)^{2}}{4 \varepsilon^{2}} \int_{-\varepsilon}^{\varepsilon} \ell\left(\tau_{t}, x\right)|x|^{2} d x \\
& \asymp \frac{(2-\delta)^{2}}{6} \ell\left(\tau_{t}, 0\right) \varepsilon \quad \text { as } \varepsilon \downarrow 0 .
\end{aligned}
$$

From (A.7), we therefore obtain

$$
\begin{aligned}
\lim _{\epsilon \downarrow 0}\langle G\rangle_{t} & =0 \quad \text { a.s., } \\
E\left[\langle G\rangle_{\Lambda(t)}\right] & =O(\varepsilon) .
\end{aligned}
$$

Equations (A.6) and (A.7) are the main parts of what we need; all that remains is to put the bits together.

So we let $M$ denote the local martingale part of $G$, fix some positive time horizon $T$ and construct the FLVR. For this, we consider a sequence $\varepsilon=2^{-n}$ of values of $\varepsilon$ and consider the portfolios $\varphi$ given by (A.5) for the different values of $\varepsilon$. We are only going to use this portfolio until the stopping time which is the minimum of $\Lambda_{T}$ and

$$
\theta_{n}:=\inf \left\{t:\left|M_{t}\right|>n^{-1}\right\},
$$

after which everything stops. Now we have (with $M_{t}^{*}:=\sup \left\{\left|M_{u}\right|: u \leq t\right\}$ )

$$
P\left[M_{\Lambda_{T} \wedge \theta_{n}}^{*}>n^{-1}\right] \leq n^{2} E\left[\left(M_{\Lambda_{T} \wedge \theta_{n}}^{*}\right)^{2}\right] \leq 4 n^{2} E\left[M_{\Lambda_{T} \wedge \theta_{n}}^{2}\right]
$$


by Doob's submartingale maximal inequality, and in view of (A.7), we have the bound

$$
P\left[M_{\theta_{n}}^{*}>n^{-1}\right] \leq C n^{2} 2^{-n}
$$

for some finite constant $C$. Hence by Borel-Cantelli, for all but finitely many $n$, we have $M_{\theta_{n}}^{*} \leq n^{-1}$ and therefore $\theta_{n}>\Lambda_{T}$. The negative part of $G_{\Lambda_{T} \wedge \theta_{n}}$ is no more than $n^{-1}$, and as we let $n \rightarrow \infty$, the terminal value $G_{\Lambda_{T} \wedge \theta_{n}}$ converges to $\mu \ell(T, 0)$, which is of course nonnegative and positive with positive probability. The FLVR is constructed.

\section{References}

1. Albrecher, H., Mayer, P., Schoutens, W., Tistaert, J.: The little Heston trap. Wilmott Magazine, 83-92 (2007)

2. Bernard, C., Cui, Z., McLeish, D.: On the martingale property in stochastic volatility models based on time-homogeneous diffusions. Math. Finance 27, 194-223 (2017)

3. Çetin, U.: Diffusion transformations, Black-Scholes equation and optimal stopping. Ann. Appl. Probab. 28, 3102-3151 (2018)

4. Clark, I.J.: Foreign Exchange Option Pricing: A Practitioner's Guide. Wiley Finance Series, New Jersey (2011)

5. Delbaen, F., Schachermayer, W.: A general version of the fundamental theorem of asset pricing. Math. Ann. 300, 463-520 (1994)

6. Delbaen, F., Schachermayer, W.: The fundamental theorem of asset pricing for unbounded stochastic processes. Math. Ann. 312, 215-250 (1998)

7. Delbaen, F., Shirakawa, H.: No arbitrage condition for positive diffusion price processes. Asia-Pac. Financ. Mark. 9, 159-168 (2002)

8. Dynkin, E.B.: Markov Processes. Springer, Berlin (1965)

9. Göing-Jaeschke, A., Yor, M.: A survey and some generalizations of Bessel processes. Bernoulli 9, 313-349 (2003)

10. Guo, Z.J.: A note on the CIR process and the existence of equivalent martingale measures. Stat. Probab. Lett. 78, 481-487 (2008)

11. Heston, S.: A closed-form solution for options with stochastic volatility with applications to bond and currency options. Rev. Financ. Stud. 6, 327-343 (1993)

12. Hulley, H., Platen, E.: A visual classification of local martingales. UTS Quantitative Research Centre Research Paper no. 238 (2008). Available online at https://doi.org/10.2139/ssrn.2175896

13. Hulley, H., Platen, E.: A visual criterion for identifying Itô diffusions as martingales or strict local martingales. In: Dalang, R., et al. (eds.) Seminar on Stochastic Analysis, Random Fields and Applications, vol. VI, pp. 147-157. Springer, Basel (2011)

14. Itô, K., Watanabe, S.: Transformation of Markov processes by multiplicative functionals. Ann. Inst. Fourier 1, 13-30 (1965)

15. Kotani, S.: On a condition that one-dimensional diffusion processes are martingales. In: Émery, M., Yor, M. (eds.) Séminaire de Probabilités XXXIX, in Memoriam Paul-André Meyer. Lecture Notes in Mathematics, vol. 1874, pp. 149-156. Springer, Berlin (2006)

16. Mijatović, J., Urusov, M.: Deterministic criteria for the absence of arbitrage in one-dimensional diffusion models. Finance Stoch. 16, 225-247 (2012)

17. Mijatović, J., Urusov, M.: On the martingale property of certain local martingales. Probab. Theory Relat. Fields 152, 1-30 (2012)

18. Palmowski, Z., Rolski, T.: A technique for exponential change of measure for Markov processes. Bernoulli 8, 767-785 (2002)

19. Revuz, D., Yor, M.: Continuous Martingales and Brownian Motion, 3rd edn. Springer, Berlin (2004)

20. Rogers, L.C.G., Williams, D.: Diffusions, Markov Processes and Martingales: Volume 1, Foundations, 2nd edn. Cambridge University Press, Cambridge (2000) 
21. Rogers, L.C.G., Williams, D.: Diffusions, Markov Processes and Martingales: Volume 2, Itô Calculus, 2nd edn. Cambridge University Press, Cambridge (2000)

22. Rouah, F.: The Heston Model and Its Extensions in Matlab and C\#. Wiley, New Jersey (2013)

23. Stroock, D.W., Varadhan, S.R.S.: Multidimensional Diffusion Processes. Springer, Berlin (1979)

24. Williams, R.J.: Introduction to the Mathematics of Finance. Am. Math. Soc., Providence (2006)

25. Wong, B., Heyde, C.C.: On changes of measure in stochastic volatility models. J. Appl. Math. Stoch. Anal. 2006, 1-13 (2006). Article ID 18130 